\title{
Real Magnetic Charges in the Substance, Ferrogravitation and Technical Levitation
}

\author{
Robert A. Sizov* \\ Solid State Physics Institute, Moscow Region, Russia \\ Email: sizov.robert@gmail.com
}

Received 12 August 2015; accepted 18 September 2015; published 21 September 2015

Copyright @ 2015 by author and Scientific Research Publishing Inc.

This work is licensed under the Creative Commons Attribution International License (CC BY). http://creativecommons.org/licenses/by/4.0/

(c) (i) 0 pen Access

\section{Abstract}

Experimental and theoretical investigations by author of the present article (period: 1968-2013) have shown that the magnetic spinor particles (magnetic charges) are real structural components of atoms and substance and are immediate sources of all magnetic fields in Nature. Joint orbital currents of electric and magnetic charges within atomic shells are the natural sources of gravitational field which is a vortex electromagnetic field. The vector character of the elementary gravitational field is similar to the vortex magnetic field that allows entering in the submission of such fundamental states of the gravitational field, as a paragravitation and ferrogravitation. Physical masses (atoms, nucleons, substance, etc.), which emit ferrogravitational field are repelled off from sources paragravitational field, for example, from Earth. It is a manifestation of the effect of levitation, which was discovered by the author of this article. Technical forces levitation, that induced by technical ferrogravitational fields, could be used in transport, lifting and space technology, power engineering and many other areas of human activity. The main reasons that real magnetic charges are "buried alive" in modern theoretical notions are as physics their of confinement in the structures of atoms and substance which are radically different from the confinement of electrons so and fallacious electromagnetic concept Maxwell in which the magnetic field is by mistake deprived of its own source of the magnetic pole or of the magnetic charge.

\section{Keywords}

Magnetic Spinor Particles (Spinors and Antispinors), Magneton and Antimagneton, Bispinor, Physical Mass, Vortical Electromagnetic (Gravitational) Field, Paragravitation and Ferrogravitation, Levitation Effect

\footnotetext{
${ }^{*}$ At present works as individual researcher, Dr. of physical and mathematical sciences.
} 


\section{Introduction in Physics Real Magnetic Charges, Gravitational Fields and Levitation Effect}

Magnetic poles (magnetic charges) as immediate sources of all magnetic fields and magnetic manifestations in the Nature were discovered by the author in structures of atoms and substance and were presented to the scientific society in 2001 in publication [1]. The results of numerous experiments conducted by the author showed that in the structures of the physical mass (atoms, nucleons, substance and other) magnetic spinor particles as spinors so and antispinors were present in approximately the same amounts as electrical particles [2]-[5]. The discovery by author of real magnetic charges as structural components of atoms and substance and the elucidation of the basic physical parameters of the magnetic spinor particles, which together with electrical particles make up the atomic shell, allowed him to offer concept of real atomic structure, as well as to clarify existing representations of physics magnetism, gravity, levitation, and some others. Most fully this theme is presented in the article the author 2015 [6], in which also contains the abstracts his major experiments related to real magnetic charges in substance.

Magnetic spinors inside the atomic shells (the author calls them magnetons) are the fundamental particles, which at its physical parameters are the magnetic analogs of electrons, that is, they have the values of the charges and spins are equal to the corresponding values for the electrons. Magnetons as electrons have a charge with a negative sign $\left(\mathrm{g}^{-}\right)$; they belong to the class of leptons and on their statistical properties to fermions (spin equal to 1/2).

Magnetic antispinors, for example antimagnetons, are true antiparticles in relation to magnetons. They have a positive magnetic charge $\left(\mathrm{g}^{+}\right)$and relate to antileptons. By their statistic properties antimagnetons are antifermions with an antispin $(-1 / 2)$ in relation to spinors. Basic physical parameters of the real magnetic spinor particles forming together with electrical particles atomic shells are presented in Appendix to the article.

Note 1. It should be added that the mere detection of magnetic spinor particles (of magnetic charges), which managed to make the author of this article should not be seen as something unique. Merit of the author is only in the fact that he showed participation of the real magnetic charges in the structures of atoms, substance and, in general, in the compositions of the Physical Mass (PM). Of course, this is a very important conclusion. This was followed by a chain of physical findings and experiments that, in the end, and led the author to his GravitoPhysics and Gravto-Technics. As for the fact of detection of real magnetic charges, then it is a merit, first of all, a wonderful Felix Ehrenhaft, who made his discoveries in the early 20th century, and his numerous followers [7]. The reasons, which more than a hundred years, block the recognition of fundamental experiments and informed conclusions F. Ehrenhaft, discussed in detail in the publications of the author and summarized in this article.

Namely magnetic bispinor (magnetic spinor pair: magneton and the corresponding him antimagneton) under rotation in the lattice conductor will be created in the plane rotation of the well-known vortex magnetic field, which is determined by the axial vector rotH, where $\mathbf{H}$ is vector of instantaneous strength of vortex magnetic field in every him point. Process of formation of the latter field is described by equation: $\mathrm{k} \operatorname{rot} \mathbf{J}_{\mathrm{g}}=\operatorname{rot} \mathbf{H}$, where $\operatorname{rot} \mathbf{J}_{\mathrm{g}}$ is the vortex current of magnetic charges and in model representation is the rotating magnetic bispinor and $\mathrm{k}$ is the proportionality factor. The role of the electric current in this process consists solely in the "organization” of the vortex movement of magnetic bispinors around lines of electric current. The process, that has been noted above, must be written in the form: $k \mathbf{J}_{\mathrm{e}}=\operatorname{rot} \mathbf{J}_{\mathrm{g}}$, where $\mathbf{J}_{\mathrm{e}}$ is vector density of the linear electric current. Thus, the sequence of the actual physical processes in a conductor with a constant electric current, which leads to the formation of a vortex magnetic field, can be written as: $\mathbf{J}_{\mathrm{e}} \rightarrow \operatorname{rot} \mathbf{J}_{\mathrm{g}} \rightarrow \operatorname{rot} \mathbf{H}$. We will notice that in Maxwell's electromagnetic (EM) concept [8], in which real magnetic charges are ignored (unintentionally), the sequence of processes at formation of a vortex magnetic field round the conductor with electric current is represented as follows: $\mathbf{J}_{\mathrm{e}} \rightarrow \operatorname{rot} \mathbf{H}$.

On the basis of his own experimental and theoretical investigations [1]-[6] the author has shown that the atomic shells, consisting of electric and magnetic spinor particles, will be electromagnetic, but not purely electronic as it is generally accepted, with the number of magnetic particles in the atoms approximately equal to the number electrical particles. Namely electromagnetic shells of the atoms are the natural sources (generators) of gravitation field, which in really is a vortex electromagnetic field. The elementary source of the gravitational field is a spinor electromagnetic quasi-particle which was given to the author's name s-graviton (s = source). In 
the composition the s-graviton: two spinors (electron and magneton) and two antispinors corresponding to them. Figuratively speaking the s-graviton is a combination of electric and magnetic bispinors rotating in antiphase on the same atomic orbit. This quasi-particle may also be presented as two joint antiphase orbital currents electric and magnetic charges. In the model representation the vortex orbital EM-current, i.e. s-graviton, must be written in the following form: $\operatorname{rot}\left[\mathbf{J}_{\mathrm{e}}-\mathbf{J}_{\mathrm{g}}\right]$, where $\mathbf{J}_{\mathrm{e}}$ and $\mathbf{J}_{\mathrm{g}}$ the vectors instantaneous density currents electric (e) and magnetic (g) charges, corresponding to their vortex (circular) flows. Then the equation of the process of gravitational field formation by s-graviton can be presented in the form:

$$
\mathrm{k} \operatorname{rot}\left[\mathbf{J}_{\mathrm{e}}-\mathbf{J}_{\mathrm{g}}\right]=\operatorname{rot}[\mathbf{E}-\mathbf{H}]
$$

where $\mathbf{E}$ and $\mathbf{H}$ are vectors of instantaneous strength of electric and magnetic fields in the composition of the vortex electromagnetic (gravitational) field. Minus signs put in the above equation of the process gravitational field formation correspond both to reciprocal antiphase of orbital currents electrical and magnetic charges so and anti-orientation of vectors of instantaneous strength of electric and magnetic fields in every point of the gravitational field. As follows from the above Equation (1), the gravitational field is a vortex electromagnetic field which is characterized by changes in the directions of the electric and magnetic fields strength vectors $(\mathbf{E}$ and $\mathbf{H})$ which are equal by absolute value and opposite directed in every point. Unlike the vortex magnetic field, each point of which corresponds to one vector of instantaneous strength $\mathbf{H}$, each point elementary gravitational field correspond to two vectors of the instantaneous strength of the fields $\mathbf{E}$ and $\mathbf{H}$, which are equal in magnitude and oriented anti-parallel to each other.

If polarization of vortex vectors $\operatorname{rot}\left[\mathbf{J}_{\mathrm{e}}-\mathbf{J}_{\mathrm{g}}\right]$ of s-gravitons is realized in the structures of physical masses (atoms, nucleons, substance, etc.), what is accompanied by polarization of vortex vectors $\operatorname{rot}[\mathbf{E}-\mathbf{H}]$, then in analogy with magnetic fields ferromagnetics, the gravitational fields being emitted by these masses can be called a ferrogravitational fields (FGF). The gravitational field formed by physical masses in the absence polarization of the vortex vectors of s-gravitons in their structures is a tensor or quasi-scalar field. And again, in analogy with magnetism, such field can to define as paragravitational field (PGF). Vector conditions corresponding to these fields are written in the following form: $<\operatorname{rot}[\mathbf{E}-\mathbf{H}]>\neq 0$ for ferrogravitational field and $<\operatorname{rot}[\mathbf{E}-\mathbf{H}]>=0$ for the field paragravitational.

Since 2001 ([1]), the author, in his publications tried to explain that all varieties of physical mass (PM) are electromagnetic structures atomic type, consisting of electric and magnetic spinor particles. One of the main characteristic properties of all varieties of physical mass is their ability to emit a gravitational field that is formed as the result of a joint orbital current of electric and magnetic charges and is the vortex electromagnetic field. Thus, the Mass can still be called as electromagnetic spinorial structure which forming a gravitational field. It is important to emphasize that PM and, for example, Matter of completely different physical categories. The physical masses are, for example, nucleons and atoms. It is important to note that the individual spinor particles, as electric so and magnetic, are massless, because the mass is the result of their joint "structural operations”. For example, the electron is massless particle and no “divine bosons” can’t give him Weight. For example, the electron is massless particle and even “divine Higgs bosons” can’t give him a Mass

The physical masses, emitting ferrogravitational field are pushed off from sources paragravitational field, for example, from the Earth. The last action is a manifestation levitation effect, which was first discovered by the author of this article and very widely distributed in Nature [9]. In principle, such effects as gravitational volatility of atoms (molecules) of light gases, for example, hydrogen and also convectional processes in liquids and gas media should be also attributed to the natural levitation manifestations. The fact of participation of the so-called gravitational levitation in the volatility of atoms (molecules) of hydrogen was detected in the experiment performed by the author of this article [10]. The levitation forces, which is caused by natural ferrogravitational fields, emitting by cells, are responsible for vertical growth (development) of plants and trees, as well as maintain them in an upright position. Namely, the forces of levitation allow Bumble bees to perform their amazing flights as well as allow of migratory birds stay in the air for a long time.

Note 2. Results of experiments author with magnetic charges and their currents in superconductors will allow him to develop technology to produce of the technical ferrogravitational field (FGF). Technical FGF and levitation forces formed by them can be used in transport, lifting and space engineering, in power engineering, as well as in numerous Gravito-Physical and Gravito-Chemical technologies (see publications author's [10] [11]). 


\section{Physical Basis and Methods of Obtaining Ferrogravitational Field}

The discovery by the author of this article levitation effect and elucidation its real physics, allowed the development of effective methods for generating artificial ferrogravitational field (FGF) and use it to get a technically significant forces of levitation. In general, the problem of creating artificial FGF consists in the organization of such physical States in the conduction band superconductors, in which is formed s-gravitons. As was shown by the author (see [1] [5]), under transition of a conductor in a superconducting state, magnetic charges, which were associated in the potential of the conduction band, going out in a free state from of interaction with the crystal lattice of the superconductor. Under this, an electric current passes through the superconductor without resistance, since the free magnetic charges, revolving around the lines of the electric current, has no "friction" upon the lattice of the superconductor.

According to the author, in present time in the oblast of experimental physics there are several ways artificial formation of s-gravitons, i.e. methods of obtaining experimental ferrogravitational field with the use of superconductors:

-using joint permanent currents of electric and magnetic charges in the superconducting solenoid [1] [5] and in superconducting ring [12];

-using a superconducting metal-ceramic disc rotating in a magnetic field [13].

It is important to note that promising for practical use suitable only developed by the author of the article creation method FGF when passing the joint permanent currents of the electric $\mathbf{J}_{\mathrm{e}}$ and magnetic $\mathbf{J}_{\mathrm{g}}$ charges through a superconducting solenoid. Currents $\mathbf{J}_{\mathrm{e}}$ and $\mathbf{J}_{\mathrm{g}}$ are obtained from relevant sources, i.e., respectively, under the action of EMF and its magnetic counterpart-magneto-motive force (MMF).

It should be noted that only the last method made it possible to provide the necessary physical and technical measures for repeated multiplication of the difference of the magnetostatic potential and the corresponding values of MMF.

In the method of the author is required to implement two different physical-technical processes:

-creation of technically significant magneto-motive force (MMF);

-implementation, using MMF, current of magnetic charges in static superconducting solenoid, which mate with a corresponding of the electrical current.

If the creation of electric current in the superconductor is not a serious problem, creation a current of magnetic charges is an extremely complex physical and technical problems and, above all, when you create a current source of magnetic charges, i.e. the formation of technically significant magneto-motive force. Thus, the works associated with obtaining the permanent current of magnetic charges accounted for the main share of human and financial efforts of the author aimed at the development and testing of the method of obtaining experimental ferrogravitational field.

Technology of obtaining of magneto-motive force based on the mechanical separation of magnetic charges in pairs of + and - in a magnetic field. As a material for the implementation of experiments on the separation of magnetic charges in pairs was chosen the lead, which is a superconductor of the first kind with the superconducting transition temperature $7.2^{\circ} \mathrm{K}$. Schematic diagram of the experiments of the author on the separation of magnetic charges in pairs of + and - in a magnetic field is presented in Figure 1.

It should be noted that obtained in these processes magnetic charges on the particles of lead are very small and require further procedures of charged enrichment. Masses of lead, charged magnetic charges of opposite signs, after the separation of charges in pairs, repeatedly passed the stage of magnetic charged enrichment (see [5]). Enrichment of the primary magnetic charged on the particles of lead were implemented by the magnetostatic interaction (repulsion or attraction) between the magnetic charges in the superconducting state of the material (lead). Experimental magnetic potential difference of the charges in vapor of leaden compacts, charged magnetic charges of opposite signs, was used to create a current of magnetic charges in a superconducting solenoid. In these experiments, by passing a joint permanent currents $\mathbf{J}_{\mathrm{e}}$ and $\mathbf{J}_{\mathrm{g}}$ was experimentally obtained ferrogravitational field [1] [5].

\section{Devices for Producing Technical Ferrogravitational Field (FG-Generators)}

The results of years of research by the author of magnetic charges and its currents allowed him to develop the principles of the device generators FGF (FG-generators) three of which are described below [10] [11]. 


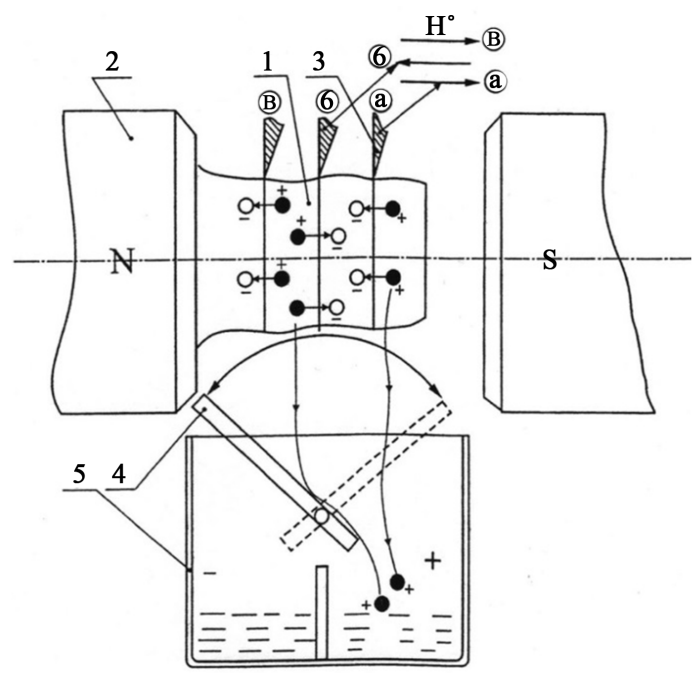

Figure 1. Scheme of the setup for primary separation of magnetic charges in pairs of + and -, as well as the separation of charged particles of lead into two groups according to the sign of their charge. Shown: trial body 1 , the poles of the electromagnet 2 , the blade of the cutting tool 3, the flap 4 of the hopper 5 . Depending on the direction of external magnetic field flap distributes the magnetic charges of the particles cut from the surface of the test body, in the relevant section of the bunker.

\subsection{Solenoidal Generator of the Ferrogravitational Field}

As part of this generator (see Figure 2): coil 1 with winding 2 of the wires in the superconducting state. From electric 3 and magnetic 4 sources in the coil at the same time served the agreed currents of electric and magnetic charges. Mutual consistency of the currents $\mathbf{J}_{\mathrm{e}}$ and $\mathbf{J}_{\mathrm{g}}$ by the force and tension is a necessary condition for the generation of effective FGF. Parts of the FG-generator, which during the generation of FGF should be in the superconducting state, are marked in Figure 2 with the dotted circle.

As shown by the experiments [1]-[5], is a very serious problem when receiving FGF from solenoidal sources is to ensure the conduction currents of magnetic charges through the superconducting contacts. It is important to emphasize that simple contact between superconductors, even under pressure, does not provide a conductivity of magnetic charges. For the implementation of the current magnetic charges through such contact is necessary for the formation of a homogeneous structure of the material in the contact zone according to the principle of through the "tunnel". Should be added that the problems with contacts, conductive currents of magnetic charges, is particularly difficult if use the so-called hard superconductors (type II superconductors).

\subsection{Solenoidal Generator of Ferrogravitational Field with Gravito-Soft Core (Ferrogravitational Analogue of the Electromagnet)}

Unlike purely solenoidal generator FGF described above and depicted in Figure 2, in the present generator used core 1 from gravito-soft material inserted in the coil (see Figure 3). Under the influence of FGF generated by the solenoid, in core implements the polarization of the elementary vortical vectors $\operatorname{rot}[\mathbf{E}-\mathbf{H}]$ atomic s-gravitons that allows multiple, compared with purely solenoidal FG-generator, to increase the density of lines (intensity) of FDF.

It should be noted that of the source FGF depicted in Figure 3, is responsible FG-generators is the so-called open type. These generators are intended for use in technical devices that require to get the maximum possible of forces levitation. For research purposes, if you want to use in experiments FGF high specific density (tension), use FG-generators of the closed type. The last generators are analogues of the classical electromagnets with the difference that the poles and the yoke made of gravito-soft materials.

If serious problems with the choice of materials for superconducting elements used in the compositions FG-generators do not exist, issues related to the production of effective gravito-soft materials, requires serious physical and technical research. Based on the results of gravitational experiments and natural phenomena 


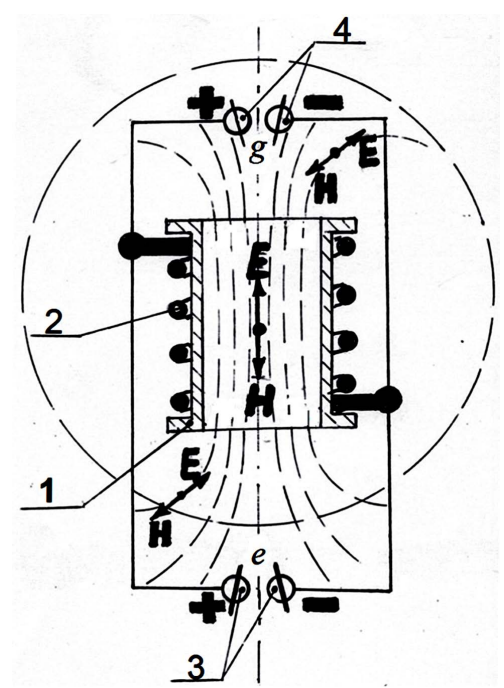

Figure 2. Diagram of a solenoidal generator of the ferrogravitational field.

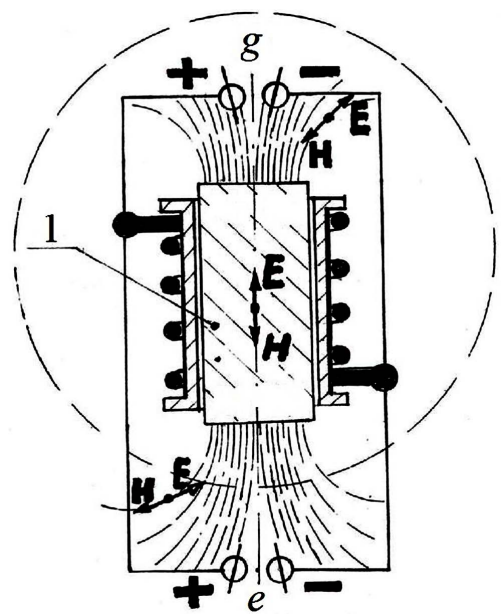

Figure 3. Scheme solenoidal generator FGF with gravito-softcore.

associated with FGF, which was considered in [9]-[11], one can expect that in the compositions of gravito-soft materials, you should use elements such as hydrogen, oxygen and carbon. A real interest in creating gravito-soft materials may present as lithium, beryllium, boron and some other so-called light elements. As for the choice of superconducting materials, the most promising are type-II superconductors. However, their use in FG-generators is complicated by the problem of the conduction currents of the magnetic charges through the superconducting contacts.

\subsection{Permanent Ferrogravitonic (Ferrogravitational Analogue of a Permanent Magnet)}

This is solid source FGF with a stable polarization of the vortex fields rot[E- $\mathbf{H}]$ atomic s-gravitons in its composition (see Figure 4). Is ferrogravitational analogue of the permanent magnet. The creation of such generators are able to produce a real energy revolution, because this will allow to directly use the power potential associated with the gravitational field of the Earth. Generation FGF by permanent ferrogravitonic can be implemented in a wide range of temperatures, e.g., above $20^{\circ} \mathrm{C}$, that will get rid of the very complex and expensive cryogenic equipment required in activity solenoidal generators FGF.

When describing solenoidal generators FGF with Gravito-soft core was given a list of items that can be used in the compositions of the gravito-soft materials. As structural components of materials when creating permanent ferrogravitonics, in addition to the above, should include the atoms stabilizers condition ferrogravitation. 


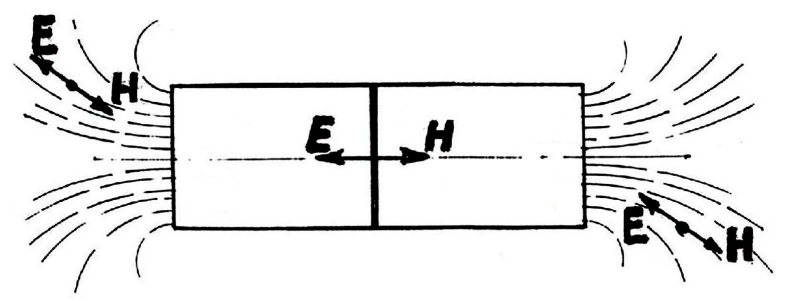

Figure 4. Scheme permanent ferrogravitonic analogue of a permanent magnet with stable polarization vortical elementary vectors of FGF emitted by the atomic shells.

\section{The Most Important Areas of Practical Application of Technical Levitation}

Earlier in this article were presented the physical basis of the processes, determining the artificial generation of FGF and proposed concepts device FG-generators. The purpose of these devices is the formation of technically significant levitation forces induced in a pair: the Earth and source of FGF. Physical masses (atoms, substance, etc.) emitting FGF will be pushed out of the Earth along the earth's vertical. The magnitudes of the levitation force depends on the density of lines (tension) of the FGF. Below, this article discusses some of the methods and devices proposed by the author for use in various spheres of engineering, with the participation of generators FGF and induced their fields of the levitation forces.

\subsection{Passenger and Freight Transport in Near-Earth Space}

Schemes gravitationally flying apparatuses (further: GFA or GF-apparatuses) used for transport purposes are shown in Figure 5. Technically sound form of such devices is a form of "plates". In their composition: gravitolifting Platform 1, containing FG-generators and cargo-passenger Deck (salon) 2. In principle, there may be various schemes of the mutual arrangement of the Platform and the Deck at GF-apparatuses, for example, with top location of the Deck relative to the Platform (Figure 5(a)) and its lower position relative to the Platform (Figure 5(b)).

FG-generators, which are used in the construction of GF-apparatuses can be both solenoidal with gravito-soft core (see Figure 3) and, what most preferably, a permanent ferrogravitonics (see Figure 4).

Given that technical forces of levitation, which is implemented in the proposed GF-apparatuses, always directed upwards, along the earthly vertical, for horizontal movement of such devices requires traditional propulsions: screw or jet 3 (shown in Figure 5). If GF-apparatuses, operated in air, in their design, can use the rudders and stabilizers provisions similar to those used in airplanes.

Overall flight GF-apparatuses are expected to be implemented at a height greater than $\approx 30 \mathrm{~km}$ from the Earth.

As already noted, vehicle capacity gravito-transport apparatuses is determined by the total density of lines (intensity) FGF emitted by the whole complex of FG-generators participating in their compositions. According to rough estimates, the hoisting capacity of the GFA with a mass equal to the mass of modern aircraft, at least not inferior to the latter. It is assumed that the use of GF-apparatuses can provide better security compared to the plane. Considering that through use of GF-apparatuses do not need a runway, fuel economy and noise reduction, the attractiveness of GF-technology is more than obvious.

\subsection{Gravito-Lifting Devices}

For these purposes, can be used as remotely controlled gravito-lifting devices so and gravito-lifting apparatuses that are controlled by pilots. Such gravito-lifting devices able to replace helicopters, and in some cases cranes.

According to their design principles gravito-lifting device similar to GF-apparatuses transport purposes.Like the latter, they include as a basis gravito-lifting platform with FG-generators. On Figure 6 in the technico-artistic form shown gravito-lifting apparatus controlled by the pilot, in the work. For horizontal movement gravitoliftting devices must use a traditional mover (probably a screw).

\subsection{Gravito-Space Technology}

As you know, the lion's share of rocket fuel used in space launches, is expended to overcome the so-called 


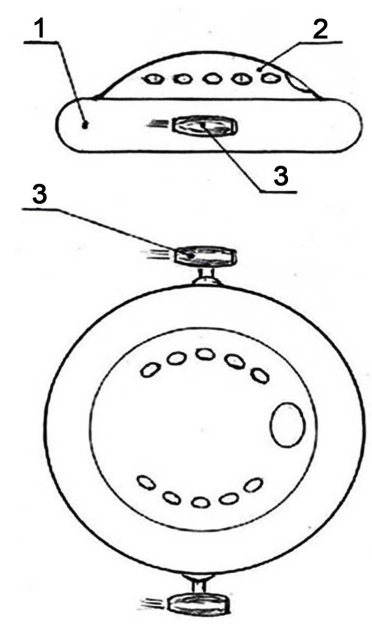

(a)

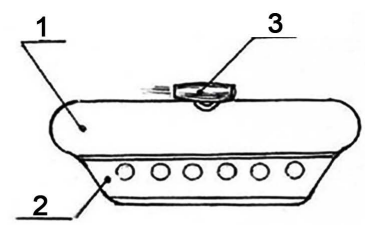

(b)

Figure 5. Schemes GF-apparatuses used in transport chains: (a) GFA with a top location of the passenger Deck; (b) with bottom location of the Deck relative to the lifting Platform.

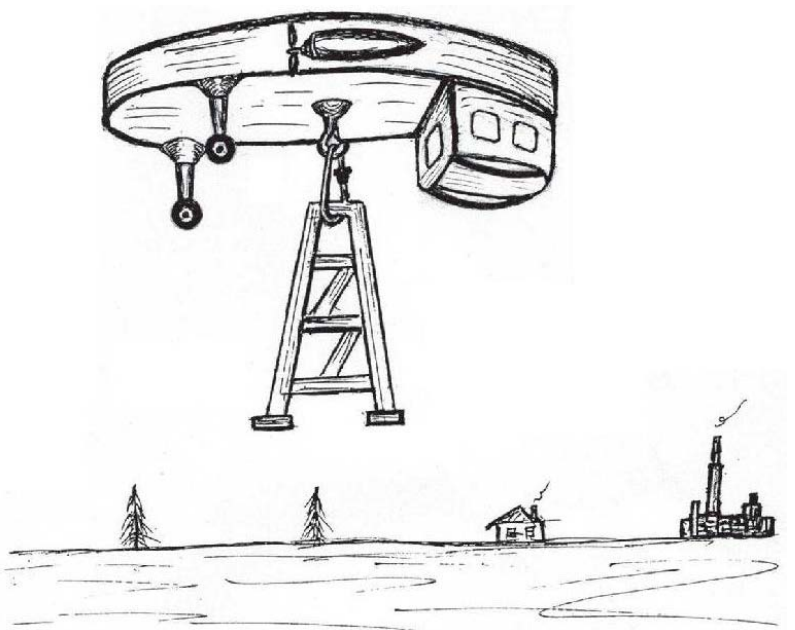

Figure 6. Gravito-lifting apparatus, controlled by the pilot, in the work.

terrestrial gravity. Use Gravito-Physical principles to exit the gravitational field of the Earth will get rid of the need to burn thousands of tons of very costly and environmentally harmful fuel in the atmosphere of our planet. However, after the release of the ship from the area of the Earth's gravitational field, for further movement in the space required, for example, the jet thrust. In principle, the device gravito-spacecraft similar to the apparatuses for flights in near-earth space. It is important to note that the Gravito-space apparatuses (GSA) are systems reusable, what distinguishes them from modern disposable rocket-space complexes. In addition, to start GSA't need spaceports, anyway, in their modern form.

To the above sleduet to add that using FG-generators possible as the creation of artificial static weightlessness so and the formation of an artificial weighting of solids and organisms. The latter will be a very important factor in the implementation of long-term space expeditions. It should also be noted that the implementation of artificial gravity will avoid possible medico-biological problems associated with the use of FG-generators in the implementation of the near-earth flights.

\subsection{Gravito-Chemical Technology}

In his publications the author repeatedly emphasizes that all physico-chemical processes in Nature, ultimately 
determined by the forces of "Dark energy" that affect as on electric and magnetic charges so and on nucleons, atoms, molecules, and others. The latter forces are induced spinor fields: electric, magnetic and electromagnetic (primarily gravitational fields) and implemented in the Power-medium, which might be called the Power-ether. As shown by the author in [14], and in this article, the gravitational fields can be paragravitatinal and ferrogravitational. Gravitational "dark energy", which is implemented, for example, in pairs of atoms or nucleons, emitting paragravitational field, determines the formation of atomic condensates (simple substances) and nucleon condensates (i.e., nuclear substance). Acting intensive FGF at real education, for example, water molecules, can expect a weakening of the bonding forces between atoms of hydrogen and oxygen. Such a scenario opens great opportunities for the use of hydrogen in the world economy, as the inexhaustible, cheap and environmentally friendly fuel. Marked by the author above example with the possible release of hydrogen from water is just one particular illustration of the chemical-technological prospects offered by Gravito-Physics in chemistry and physical-chemical technologies.

\subsection{Gravito-Power Engineering}

Levitation forces, that induce technical FG-generators, can be used in the energy sector, as well as in engines of moving vehicles. Using simple technical solutions levitation forces can be used to rotate the shafts of electric generators and motors of different vehicles. Of course, these brighter prospects may be opened to humanity in that case that will be developed and applied practically permanent ferrogravitonics.

In addition, when exposed to intense ferrogravitational field on real physical objects, for example, molecular compounds, can significantly change the magnitude of the forces of interatomic bonds, which will lead to "life" such scientific and technical directions, as gravito-Chemistry, gravito-Biology and even gravito-Medicine.FGF is able to provide effective assistance in the development of new drugs, as well as productive varieties of crops. Can expect large positive events when using high tension FGF in sanitation and traumatology.

Of course, the list of suspected applications Gravito-Physical technologies can be quite long. The real state of Affairs in this field will be set only after creating technical FG-generators and conducting with their help, special studies.

\section{Conclusions}

In their publications (see [6] [9] [10]), the author repeatedly emphasizes that levitation effect and, as a consequence, the advanced capabilities of its practical (technical) use of more than one hundred years, hampered by high physical science. The reason for this is that physical science is unwilling to accept the possibility and even the facts of the existence of a real magnetic spinor particles (magnetic charges), which are structural components of atoms and substance. As noted above, the main reason that was delayed for 140 years the official recognition of magnetic charges, is fundamental differences in the physics of their confinement in atoms and the substance from the confinement of electrons. A significant negative factor in the recognition of the real magnetic charges in physical science, was erroneous electromagnetic concept of Maxwell, which was formed as a result of the primitive-superficial analysis of the famous experiment of Oersted. In the framework of this concept, in the absence of any real knowledge about the physical processes occurring in the conductor with an electric current, physical theory deprived magnetic field own source: the magnetic poles or magnetic charge. Namely, under the influence of the erroneous electromagnetic concept Maxwell magnetic charges "buried alive.” For this reason, a magnetic field, which by its nature was a classical spinor field turned into a physical theory in emanations or "miscarriage" of electricity.

Namely, for the above reasons, outside of the official physical representations turned out to be the real atomic structure, electromagnetic vortex nature of the gravitational field, a para- and ferrogravitation, as well as the physical essence of mass. The author shows that all varieties of physical mass (atoms, nucleons, substance, etc.) are electromagnetic structure atomic type, consisting of the real electric and magnetic spinor particles. If we mentally remove magnetic charges from the structures of the physical mass, on this "place" will only electrically particles, which themselves are not capable of forming a gravitational field, since they are only capable of emitting an electric field. Fact ignoring, for over a hundred years, of real magnetic charges of physical science, is the main reason that has not been mastered technical levitation forces and has not been used in practice gigantic energy potential associated with the gravitational field of the Earth.

Research real fundamental magnetic particles, performed by the author showed that manipulate with real 
magnetic charges, so as was customary in physics and engineering with electric particles, for example, such as electrons, very difficult. However, nothing prevents to work with real magnetic poles inside the substance. So you can charge the body of magnetic charges of opposite sign, enrich charge, to create currents of magnetic charges, which can only be realized in superconductors. During mating currents, magnetic and electric charges in the superconductor can be obtained experimental and in the future, and technically significant of gravitational fields. For that would implement this in practice should: desire, financial opportunities and, of course, serious experimental and technical activity. It should be noted that the magnitude of effects that can be achieved in the early stages of working with magnetic charges and magnetic currents, is quite modest. But we could remember a relatively recent history of nuclear power in the early stages of development which was not observed grandiose effects.

Note 3. Base provisions of general physics with real magnetic charges, developed by the author, were presented at the international PIERS 2009 Moscow Conference (Progress in Electromagnetics Research Symposium [15] [16]).

\section{Acknowledgements}

The author is Grateful to technical specialists: Davydov A.A., Koshelev N.V., Lomakin A.I., Moiseenkov E.V. and Staritsin S.E. which provide technical support for the main part of his experiments, concerned the researches of magnetic charges.

\section{References}

[1] Sizov, R.A. (2001) New Presentation of Nature Magnetism, Gravitation and Nuclear Forces of Bonding (Experiments and Theory). Akademizdat Center "Science", Moscow.

[2] Sizov, R.A. (1971) Journal of Experimental and Theoretical Physics, 60, 1363-1370.

[3] Sizov, R.A. (2005) Magnetic Elementary Particles as Stable Structural Components of Atoms and Substance (Experiments and Theory). Akademizdat Center "Science", Moscow.

[4] Sizov, R.A. (2007) Magnetic Fundamental Particles (Magnetons) in Physics of Magnetic and Electromagnetic Effects (Experiments and Theory). Akademizdat Center "Science", Moscow.

[5] Sizov, R.A. (2008) Electric and Magnetic Spinorial Particles as Structure-Forming Components of Mass and Electromagnetic Source Gravitation (Experiments and Theory). Akademizdat Center "Science”, Moscow.

[6] Sizov, R.A. (2015) Journal of Modern Physics, 6, 1013-1022. http://dx.doi.org/10.4236/jmp.2015.68106

[7] Ehrenhaft, F. (1910) Wiener Berichte, 119, 836.

[8] Maxwell, J.C. (1873) Treatise on Electricity and Magnetism. Vol. 1-2, Oxford.

[9] Sizov, R.A. (2012) Levitation as a Spread Force Manifestation of Natural Ferrogravitation. Akademizdat Center "Science", Moscow.

[10] Sizov, R.A. (2013) Transference of People and Loads in the Terrestrial Space by Means of Technical Ferrogravitation. Akademizdat Center "Science”, Moscow.

[11] Sizov, R.A. (2013) Generation and Technical Application of Ferrogravitation (the Collection of the Invention of the Author in Area the Gravitophysical and Gravitochemical Technologies). Akademizdat Center “Science”, Moscow.

[12] Podkletnov, E. and Nieminen, R. (1992) Physica C, 203, 441-444. http://dx.doi.org/10.1016/0921-4534(92)90055-H

[13] Tajmar, M. and de Matos, C.J. (2003) Physica C, 385, 551-554. http://dx.doi.org/10.1016/S0921-4534(02)02305-5

[14] Sizov, R.A. (2011/2012) Matter, Antimatter and Energo-Medium-Physical Triad of the Real World. Akademizdat Center "Science”, Moscow.

[15] Sizov R.A. (2009) Magnetic Particles (Magnetons)—Structural Components of Atoms and Substance, Immediate Sources of Magnetic Field. Theory and Experiments (The Report at PIERS 2009 Moscow).

[16] Sizov R.A. (2009) Electric and Magnetic Spinor Particles-The Electromagnetic Source of Gravitation, Theory and Experiments (The Report at PIERS 2009 Moscow). 


\section{Appendix}

The Main Physical Parameters of Magnetic Spinorial Particles Forming Together with Electrical Particles the Atomic Shells

Charge: The magneton (magnetic spinor) and the antimgneton (magnetic antispinor) have a magnetic charges $\mathrm{g}$ the value of which is equal to the value of the electron charge $(\mathrm{g}=\mathrm{e})$. Researches of the author showed that the greatest possibility charge of a fundamental magnetic spinor particle is equal to electron charge, i.e. gmax = e.

Sign of the charges: Like an electron a magneton has a charged with the sign "minus" $\left(\mathrm{g}^{-}\right)$. Antimagneton has a positive magnetic charge $\left(\mathrm{g}^{+}\right)$.

Mass: Like an electron a magneton andantimagneton are a massless particles, because the Physical Mass is result of joint structural "activity" of electric and magnetic spinor particles.

Class of elementary particles: The magneton is a lepton and the antimagneton-an antilepton.

Statistical properties: The magneton is a fermion (spin equal $1 / 2$ ) and the antimagneton-an antifermion (spin equal $-1 / 2$ ). 